Journal of Engineering and Applied Sciences 15 (2): 559-566, 2020

ISSN: 1816-949X

(C) Medwell Journals, 2020

\title{
Spectrum Detection Scenario over Rayleigh Channel for Cognitive Radio
}

\author{
${ }^{1}$ Oualid Khatbi, ${ }^{1}$ Ahmed Mouhsen, ${ }^{2}$ Younes Chihab and ${ }^{3}$ Zakaria Hachkar \\ ${ }^{1}$ Ingenierie Mecanique, Management Industriel et Innovation (IMMII), Universite Hassan 1er, \\ Faculte des Sciences et Techniques, BP 577, 26000 Settat, Morocco \\ ${ }^{2}$ Department of Computer Sciences, Superior School of Technology, \\ Ibn Toufail University, Kenitra, Morocco \\ ${ }^{3}$ Safi Poly disciplinary Faculty, MSISM team, Safi, Morocco
}

\begin{abstract}
The aim of this study is to investigated the performance of the energy detection in Rayleigh fading channel. Only single user detection situation was investigated. To design a communication link that models fading, we simulate the Rayleigh channel using the correlation matching criterion. After that, we check the quality of the transmission, the BER was analyzed for different modulations schemes. We found that BPSK modulation is less affected by errors. The numerical results of the detection are plotted as Pf Vs Pd for different values of SNR on the one hand and on the other hand depending on the number of samples used for the listening of the channel. The results show that the performance of ED depends on the number of samples and the SNR values.
\end{abstract}

Key words: Cognitive radio, spectrum sensing, rayleigh fading channels, energy detection, BER, performance

\section{INTRODUCTION}

Following the vertiginous developments recorded in wireless communications, the demand for spectrum has seen a remarkable increase. The Federal Communications Commission (FCC) stated that some allocated frequency bands are largely unoccupied (underutilized) most of the time (FCC., 2002). Cognitive radio is an innovative solution to meet the growing needs of wireless communication. The CR is a radio that able to alter its transmitter parameters based on interaction with its environment (Sun et al., 2013). The CR enables to Dynamically Access to Spectrum (DSA). This new paradigm will obviously involve an innovative spectral management with new functions, where the most important one and one that plays a major role in the management of the transmission mode recommended by the 802.22 standard, it's the true detection of the bands.

Many spectrum detection techniques have been proposed so far, for example the matched filter, cyclostationary and Energy Detection (ED) (Atapattu et al., 2014). In (Yucek and Arslan, 2009), different spectrum detection techniques have been presented. It is noted that special attention has been paid to blind detection methods, i.e., those that do not require information on source signals and propagation channels.

In Zeng et al. (2010) the authors analyze the probability of missed detection of ED under Nakagami endangered channels. However, the behavior of the channel influences the operation and the performance of a CR, it is wise to evaluate the performance of CR system under different channel models. In Atapattu et al. (2011), the performance of ED is studied under very low levels of SNR. The analysis relates to the derivation for the average missed-detection probability of detection in the Rayleigh channels and the nakagami-m fading channels.

We recall that the term fading refers to the temporal variation of power of the received signal caused by changes in the average transmission or path (Junior et al., 2013). Indeed, during their travelling, the signals are affected by the objects such as building, trees and so on. It seems appropriate to take care of, the two main factors that influence the spectral detection namely: fading and noise.

The model proposed by Jakes is the most widely used to characterize the fading phenomenon and more particularly the Rayleigh Model (Lee et al., 2009). The first approximation that has been used is the sum of sinusoidal method proposed by Jakes (Lee et al., 2009). We also note that, fading process can be achieved by passing complex Gaussian noise through an ARMA filter (Venkateswarlu and Sastry, 2014) or by using algorithms applying IDFT (Young and Beaulieu, 2000).

It is clear that fading contributed in a special way to impairments that affect communication systems. Therefore, there is a demand for techniques that can improve the performance of the channel. Diversity can notoriously be seen as a solution because it produces some redundancy in the received signal. The coding may also insert a redundancy in the transmitted signal

Corresponding Author: Oualid Khatbi, Ingenierie Mecanique, Management Industriel et Innovation (IMMII), Universite Hassan 1er, Faculte des Sciences et Techniques, BP 577, 26000 Settat, Morocco 
(De Alencar et al., 2014). Therefore, by simulating the performance of the channel considered with the support of certain modulation techniques and which will be the subject of a paragraph in our present work we seem a step which must precede the study of the spectral detection and in particularly in the case of CR. Given the importance of the channel in any transmission, we will begin our article by simulating the Rayleigh channel which is perfectly recommended in the literature to model several types of fading. Once the time-varying multipath transmission channel model is built, we analyze the transmission performance according to the modulations (BPSK, QPSK and 16-QAM), the analysis carried out in our case focuses on the probability of Bit Error Rate (BER) depending on the ratio received Signal on Average Noise (SNR) (Tlili et al., 1999). Finally, once the performances are evaluated, we implement under MATLAB the ED using on the one hand the model of Rayleigh built and on the other hand the modulation which realizes the best BER.

Generaly the performance of ED can be characterized by the $P_{f}$ (probability of false alarm), $P_{m}$ (probability of miss detection) and $P_{d}$ (the probability of detection). The term $\mathrm{P}_{\mathrm{f}}$ is the probability that a Secondary User (SU) decides the Primary User (PU) is active when the PU is actually inactive.what can be illustrated by the Receiver Operating Characteristics (ROC), curve which is a plot of $P_{d}$ versus $P_{f}$ or $P_{m}$ versus $P_{f}$ (Atapattu et al., 2009).

\section{MATERIALS AND METHODS}

Spectrum sensing techniques: Spectrum detection is the main operation in Cognitive Radio (CR) to detect unwanted or underutilized bands. Existing spectrum sensing techniques can be divided into three types (Cabric et al., 2004): energy detection, matched filter detection and cyclostationary detection. The energy detector does not require knowledge of the properties of the transmitted signal or channel information.

Energy detection uses the energy spectra of the received signal in order to identify the frequency locations of the transmitted signal. The spectrum sensing, in a simple form can be formulated as a binary hypothesis testing problem:

$$
x(k)=\left\{\begin{array}{c}
n(k), \quad H_{0} \\
h(k) s(k)+n(k), \quad H_{1}, k=1, \ldots, N
\end{array}\right.
$$

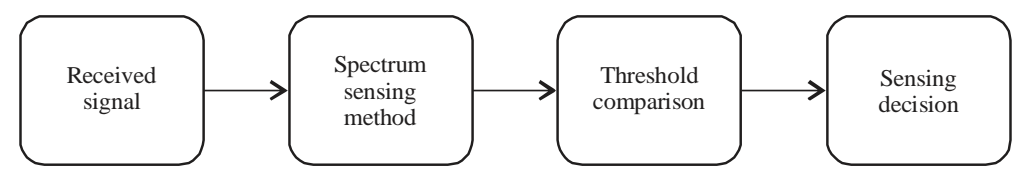

Fig. 1: General model of spectrum sensing where, $\mathrm{H}_{0}$ is the hypothesis of the absence (vacant channel) whereas the hypothesis $\mathrm{H}_{1}$ denotes the presence (occupied channel) of the PU's signal. The samples $n(k)$ are assumed to be zero-mean Gaussian random variables with variance $\mathrm{N}_{0} \mathrm{~W}$ where $\mathrm{W}$ and $\mathrm{N}_{0}$ denoting the single-sided signal bandwidth and a single-sided noise power spectral density, respectively, $h$ is the complex channel gain of the sensing channel (Nallagonda et al., 2011). The received signal is first pre-filtered by a bandpass filter to eliminate out-of-band signals. The transfer function of such a filter is given by:

$$
H(f)=\left\{\begin{array}{l}
\frac{2}{\sqrt{N_{01}}}\left|f-f_{c}\right| \leq W \\
0 \quad\left|f-f_{c}\right|>W
\end{array}\right.
$$

Then the received signal is passed through the following blocks as shown in the block diagram Fig. 1. This model is used for all detection techniques. It is enough for each technique to change the spectrum detection method block. The decision statistic of an energy ED can be calculated from the squared magnitude of the FFT averaged over $\mathrm{N}$ samples. In order to decide whether the observation $\mathrm{x}$ is generated under hypothesis $\mathrm{H}_{0}$ or hypothesis $\mathrm{H}_{1}$, it is typically accomplished by firstly forming a test statistic $\mathrm{T}(\mathrm{x})$ with the received data $\mathrm{x}$ according to different spectrum sensing algorithms, the assumption is that with the presence of a PU in the channel, there would be significantly more energy than if there was no signal present. Therefore, energy detection implies the application of a threshold $\lambda$ which is used to decide whether a transmission is present at a specific frequency, the decision rule is as follow:

$$
\mathrm{T}(\mathrm{x}) \underset{\mathrm{H}_{0}}{\stackrel{\mathrm{H}_{1}}{\rightleftharpoons}} \lambda
$$

In order to characterize the performance of spectrum detection some indicators are considered: the probability of detection, $P_{d}$ and the probability of false alarm, $P_{f} . P_{d}$ is the probability that the $\mathrm{SU}$ declares the presence of a signal PU. Great is $P_{d}$, better is the PU protection. $P_{f}$ is the probability that SU declares the presence of PU when the spectrum is unoccupied. To make the technique efficient it would be trivial to minimize this probabilistic measure:

$$
\begin{gathered}
\mathrm{Pd}=\mathrm{P}(\mathrm{T}</ \mathrm{H} 0) \\
\mathrm{P}(\mathrm{fa})=\mathrm{P}(\mathrm{T}>/ \mathrm{H} 1)
\end{gathered}
$$




\section{Probability detection over channels}

AWGN channel: In an AWGN channel and in the absence of fading, the parameter $\mathrm{h}$ in Eq. 1 is equal to one and as a consequence, the probability of detection and the probability of false alarm are given by the following Eq. 24:

$$
\begin{gathered}
\mathrm{P}_{\mathrm{d}}=\mathrm{P}\left(\mathrm{T}(\mathrm{x})>\lambda / \mathrm{H}_{1}\right)=\mathrm{Q}_{\mathrm{m}}(\sqrt{2 \gamma}, \lambda) \\
\mathrm{P}_{\mathrm{f}}=\mathrm{P}\left(\mathrm{T}(\mathrm{x})>\lambda / \mathrm{H}_{0}\right)=\frac{\Gamma\left(\mathrm{m}, \frac{\lambda}{2}\right)}{\Gamma(\mathrm{m})}
\end{gathered}
$$

where, $\lambda$ denotes the energy threshold. $\Gamma($.$) and \Gamma(.,$.$) are$ complete and incomplete gamma functions, respectively and (.,.) $\mathrm{Q}_{\mathrm{m}}$ is the generalized Marcum Q-function, $\gamma$ denote the SNR. If the signal power is unknown, we can first set the false alarm probability $P_{f}$ to a specific constant. By Eq 7, the detection threshold $\lambda$ can be determined. Then, for the fixed number of samples 2TW the detection probability $\mathrm{Pd}$ can be evaluated by substituting the $\lambda$ in Eq. 6 .

Rayleigh fading channel: When the environment is characterized by fading, h varies. In this case Eq. 4 gives the probability of detection as a function of the instantaneous SNR $\gamma$. The average probability of detection Pd may be derived by averaging (Eq. 4) over fading statistics (Mfeze and Tonye, 2015):

$$
P_{d}=\int Q_{m}(\sqrt{2 \gamma}, \sqrt{\lambda}) f_{\gamma}(x) d x
$$

where, $\mathrm{f}_{\gamma}$ is the Probability Distribution Function (PDF) of SNR under fading. If the signal amplitude follows a Rayleigh distribution, then the SNR $\gamma$ follows an exponential PDF given by:

$$
\mathrm{f}(\gamma)=\underset{\gamma}{\frac{1}{\gamma}} \exp \left(-\frac{\gamma}{\gamma}\right), \gamma \geq 0
$$

where, $\bar{\gamma}$ is the average SNR per bit. In this case, a closed-form formula for $\mathrm{P}_{\mathrm{d}}$ may be obtained (after some manipulation) by substituting $\mathrm{f}$ in Eq. 8:

$$
\mathrm{P}_{\text {dRay }}=\mathrm{e}^{-\frac{\lambda}{2} \sum_{\mathrm{k}=0}^{\mathrm{m}-2}} \frac{1}{\mathrm{k} !}\left(\frac{\lambda}{2}\right)^{\mathrm{k}}+\left(\frac{1+\bar{\gamma}}{\bar{\gamma}}\right)^{\mathrm{m}-1} \times\left(\mathrm{e}^{-\frac{\lambda}{2(1+\bar{\gamma}}}-\mathrm{e}^{-\frac{\lambda}{2} \sum_{\mathrm{k}=0}^{\mathrm{m}-2} \frac{1}{\mathrm{k} !}\left(\frac{\lambda \bar{\lambda}}{2(1+\bar{\gamma})}\right)}\right.
$$

where, $m$ denote degree of freedom. While a channel simulator is an essential component in the development and accurate performance evaluation of any communication system, we found it interesting to generate the Rayleigh distribution using a highly efficient approximation of generating a correlated Rayleigh fading sequence which has been developed by Young and
Beaulieu (De Alencar et al., 2014) in this study, the channel is approximated by a first-order autoregressive AR model. The common method used in the literature to estimate the parameter of the AR Model is based on a Correlation Matching (CM) criterion (Barbieri et al., 2009).

In order to verify that our application of the Jakes method is correct, we simulated the Rayleigh channel and compared it to the one obtained theoretically. The verification was performed by calculating the autocorrelation function (ACF) of the two signals. It is clear from the Fig. 2 that the ACFs of the two techniques (theoretical and simulated) are very similar. It should be noted that our results are comparable to those obtained by Anonymous (2019).

\section{Modulation and quality of the channel}

Modulations: The BPSK is one of the simplest forms of modulation, it's used to represent 2 bits, namely, 1 and 0 . The carrier wave is reversed by a phase of $180^{\circ}$ to represent a change in bits which is either 1 and 0 . BPSK is very effective and robust against noise especially in low data rate applications but it can modulate only 1 bit per symbol. Trivially, this is not the only possibile modulation. Another option is Quadrature Phase Shift Keying (QPSK). Even if it is not suitable for applications requiring flow. The BPSK is more robust and covers a higher distance compared to QPSK modulation (Sklar and Ray, 2009). Both BPSK and QPSK use phase shifts to separate the symbols. This produces constellations with good separation up through four symbols but beyond that better schemes are possible. The most popular of these other schemes is Quadrature Amplitute Modulation (QAM) where the symbols are placed on a grid which is centered around the origin.

All these modulation strategies and many more are used to allow coexistence between the CR-based rental system and the licensed system (Budiarjo et al., 2008). QAM is the most used modulation technique in OFDM. As a multi-level modulation scheme, QAM modulation scheme acquires higher data rate that is higher bandwidth efficiency, by sacrificing power utilization (Du et al., 2006).

Bit error rate: In any communication system we will seek to receive the data with a minimum of errors because on the one hand these degrade the performance of the system and on the other hand the quality of service is greatly affected. Bit Error Rate (BER) is an important factor that determines the performance of different modulation techniques In this section, we present BER expressions both for AWGN channels and for the Rayleigh channel for the various modulations used in this work. 


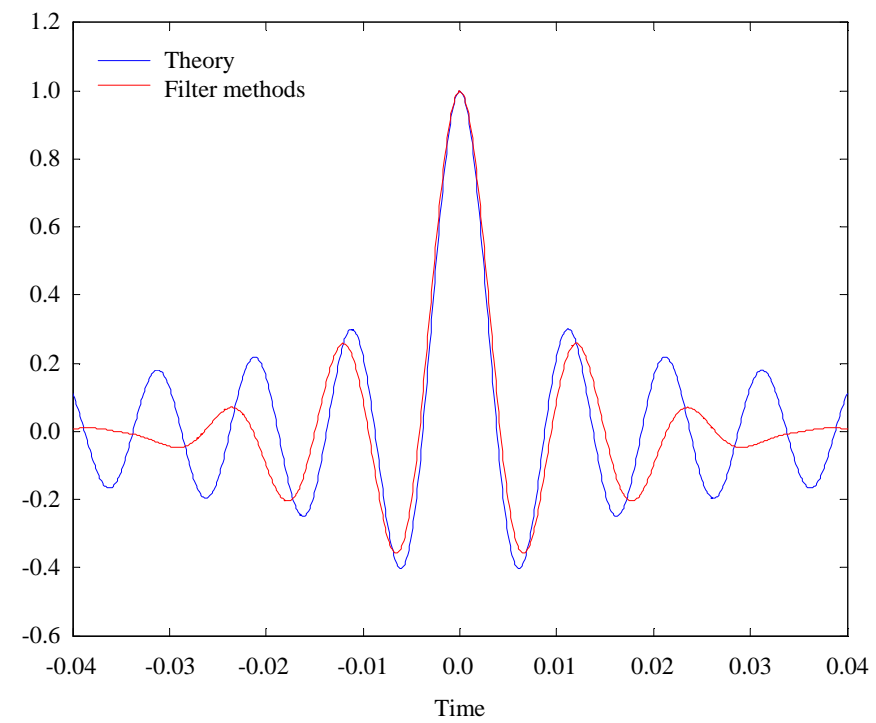

Fig. 2: Autocorelation function for rayleigh channel

The BER performance of any digital modulation scheme in a slow flat fading channel can be evaluated by the formula (Yelizati 2016):

$$
\mathrm{P}_{\mathrm{b}}=\int_{0}^{\infty} \mathrm{P}_{(\mathrm{b}, \mathrm{AWGN})(\gamma) \mathrm{P}_{\mathrm{d}} \mathrm{f}(\gamma)} \mathrm{d} \gamma
$$

where, $\mathrm{P}_{\mathrm{b}, \mathrm{AWGN}}(\gamma)$, design the probability of error of a particular modulation scheme in AWGN channel at a specific SNR:

$$
\gamma=h^{2} \frac{E_{b}}{N_{0}}
$$

where, the random variable $h$ is the channel gain, $E_{b} / N_{0}$ is the ratio of bit energy to noise power density in non-fading AWGN channel, the random variable $h^{2}$ represents the instantaneous power of the fading channel and $\mathrm{P}_{\mathrm{df}}(\gamma)$ is the probability density function of $\gamma$ due to the fading channel.

For simplicity and practical implementation, we use the BER given in the following In the AWGN channel, BER performance for BPSK can be expressed as follows:

$$
\mathrm{BER}=\frac{1}{2} \operatorname{Erfc}\left(\sqrt{\frac{\mathrm{E}_{\mathrm{b}}}{\mathrm{N}_{0}}}\right)
$$

where, $E_{b} / N_{0}$ is the ratio of bit energy to noise power density in a non fading AWGN channel, erfcis the complementary error function defined as:

$$
\operatorname{Erfc}(z)=\frac{2}{\sqrt{\pi}} \int_{z}^{\infty} \mathrm{e}^{-t^{2}} \mathrm{dt}
$$

The BER for Rayleigh fading channels can be expressed as follows:

$$
\operatorname{BER}=\frac{1}{2}\left(1-\sqrt{\frac{\frac{\mathrm{E}_{\mathrm{b}}}{\mathrm{N}_{0}}}{1+\frac{\mathrm{E}_{\mathrm{b}}}{\mathrm{N}_{0}}}}\right)
$$

In the case of Quadrature amplitude modulation $\operatorname{MQAM}(M=4,8,16)$ to compute channel's performance of both AWGN channel and rayleigh FADING channel we use the following equation:

$$
\mathrm{BER}_{\mathrm{MQAM}, A W G N}=\frac{4}{\log _{2}(\mathrm{M})}\left(1-\frac{1}{\sqrt{\mathrm{M}}}\right) \sum_{\mathrm{i}=1}^{\frac{\sqrt{\mathrm{M}}}{2}} \mathrm{Q}\left(\sqrt{\frac{3 \log _{2} \mathrm{ME}_{\mathrm{b}}}{(\mathrm{M}-1) \mathrm{N}_{0}}}\right)
$$

In Fig. 3 and for the BPSK type modulation, we have shown the evolution of BER as a function of SNR for different channels. By analyzing the obtained curves, it is deduced that the better performances are obtained on a Gaussian channel, in the absence of fading. On the other hand, the performances deteriorate enormously on the Rayleigh channel. It should be noted that we did not use BPSK modulation because: the BERs of BPSK and QPSK coincide because QPSK can in fact be seen as two coherent binary PSK systems operating in parallel and using two carriers in quadrature phase 


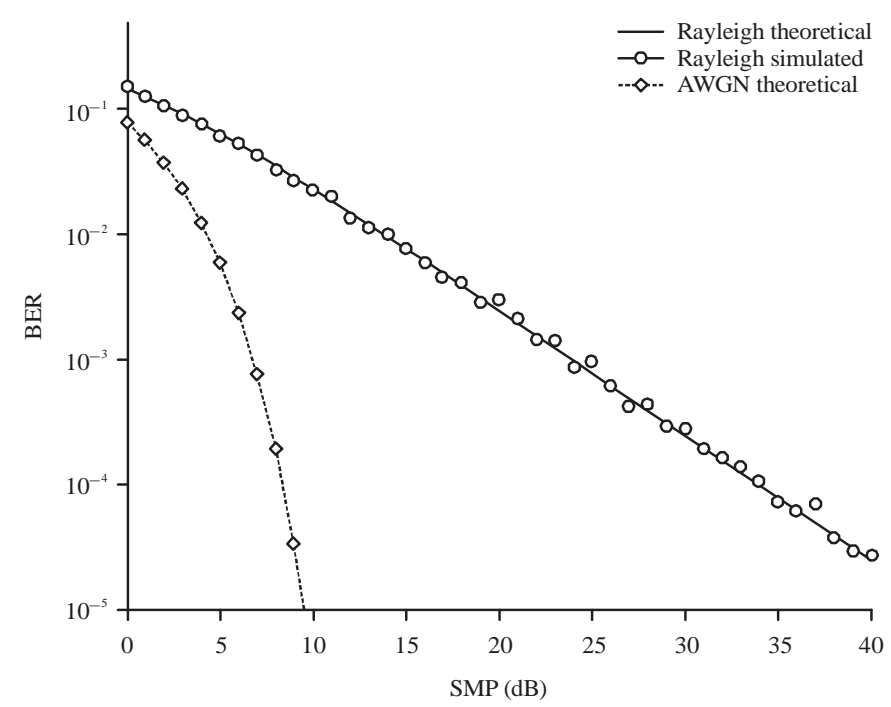

Fig. 3: Evolution of BER according to SNR (QPSK)

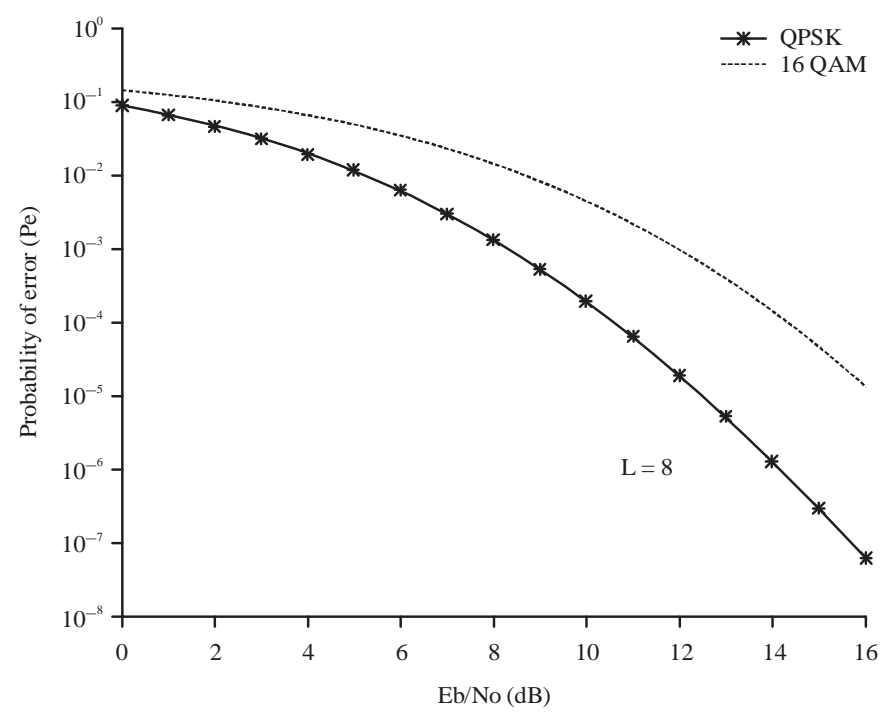

Fig. 4: Performance of QPSK and 16QAM in Rayleigh channel

(Yelizati, 2016). From Fig. 3 we also see that BER is inversely related to SNR that is high BER causes low SNR. High BER causes increases packet loss, increase in delay and decreases throughput.

For a Rayleigh channel (Fig. 4), we have represented the evolution of the error probability as a function of the quotient $\mathrm{E}_{\mathrm{b}} / \mathrm{N}_{0}$ for the two modulations QPSK and 16 QAM. The performances of the two mudulations are simulated using MATLAB function 'berfading'.

Figure 4 shows that to obtain a BER of $10^{-3}$ in Rayleigh slow fading channel. Using QPSK, requires roughly an value of $8 \mathrm{~dB}$ in terme of $\mathrm{E}_{\mathrm{b}} / \mathrm{N}_{0}$ whereas the 16 QAM modulation will requires a value of about $12 \mathrm{~dB}$. We deduce that the degradations due to fading reach more 16 QAM modulation. In terms of recall, for a fixed $\mathrm{E}_{\mathrm{b}} / \mathrm{N}_{0}$, an acceptable BER is possible with the channel coding. This can be achieved by using recoding techniques among other things.

\section{RESULTS AND DISCUSSION}

The simulation under Rayleigh channel of Pd is studied in order to find the performance of spectrum detection methods using energy as a parameter (ED), to do this in the case of CR, we consider two main assumptions: first the primary signal is deterministic and Binal Phase Shift Keying (BPSK), second the Noise is real Gaussian with mean 0 and variance 1.

After checking the modulation that responds to well-defined constraints in terms of BER (paragraph 4), we adopt the BPSK modulation under a ralyeigh flat 


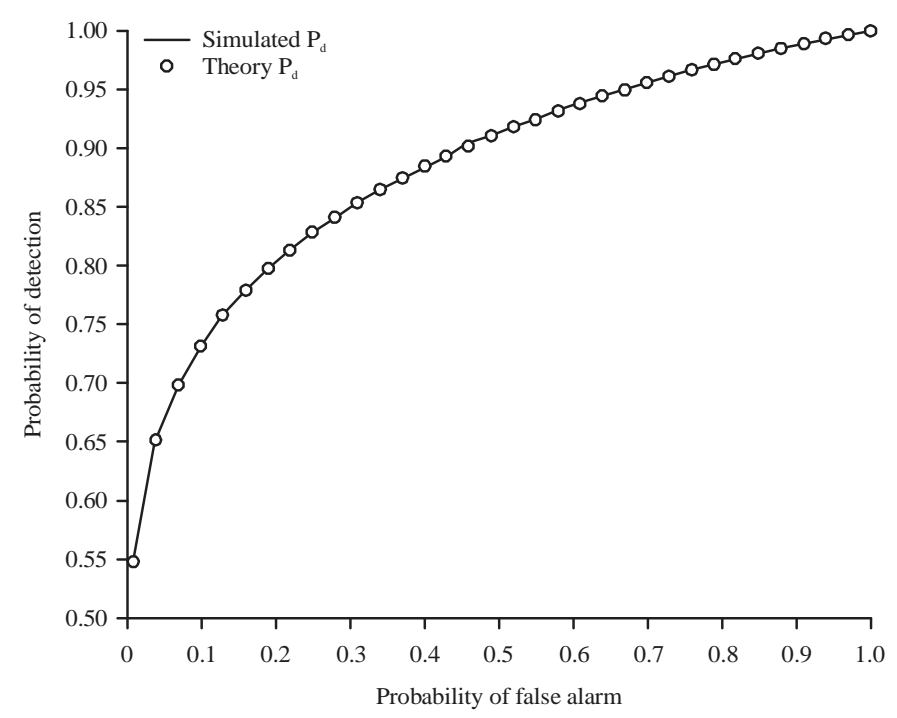

Fig. 5: Comparison betwen thorical and simulated COR

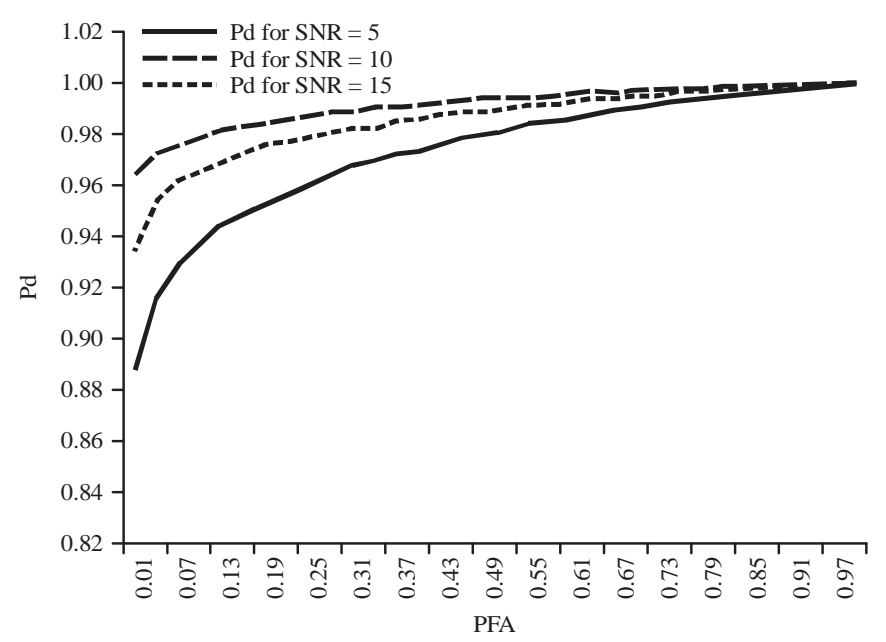

Fig. 6: ROC for ED using great SNR values

fading channel, the metric COR will be chosen as the performance indicator. Then for $L=6$, while $L$ stands for Number of sensing samples and for a signal-to-noise level of 5. As can be seen Fig. 5, the two curves: simulated and theoretical using the aforementioned performance indicator are similar, if we fix a detection value of the order of 0.9 , the corresponding threshold is of the order of 5. One can also see clearly that the probability of simulated detection remains an increasing function of the probability of false alarm.

We know that, ED implemented in the case of the AWGN channel records very good performance for high SNR values. So, varying the value of the SNR to see the effect this can have on the detection capacity. We set the number of samples involved in the process of sounding the channel at 6. As can be seen, Fig. 6, shows a performance of energy Detector under Rayleigh flat fading channel with SNR $=5,10,15$. For false alarm probability (indicating the level of undetected holes in the spectrum) of $<0.1$, the detector records quite significant performance.

We note, however, that to protect licensed transmissions, the 802.22 standard requires spectral detection at very low SNR levels. We note, for example, the IEEE 802.22 WRAN standard which requires reliable primary detection at low SNR levels, e.g., SNR at -20 dB (Atapattu et al., 2014).

Now, varying the SNR levels towards low values to see the effect of such a reduction on the detection capacity. We will fix the number of samples to 6 and we 


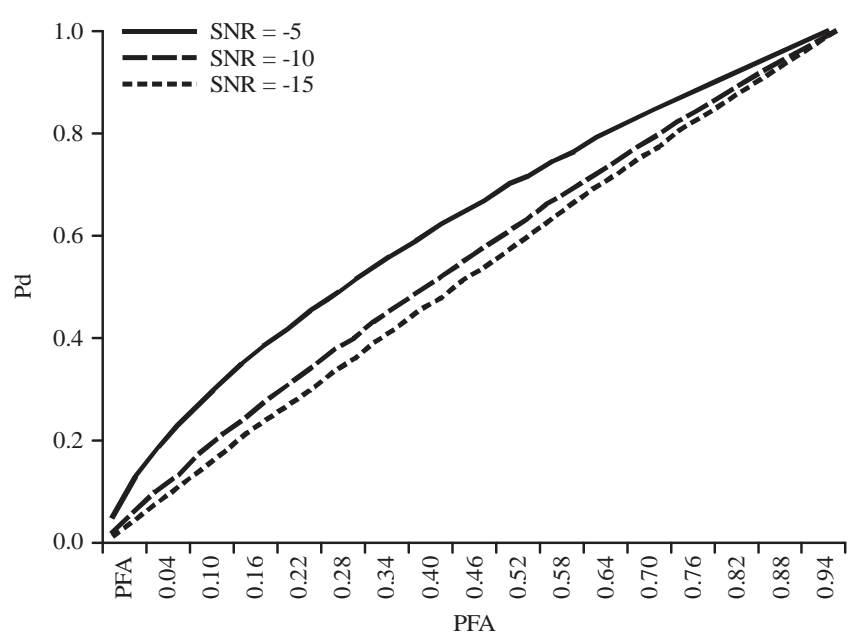

Fig. 7: ROC for various low SNR

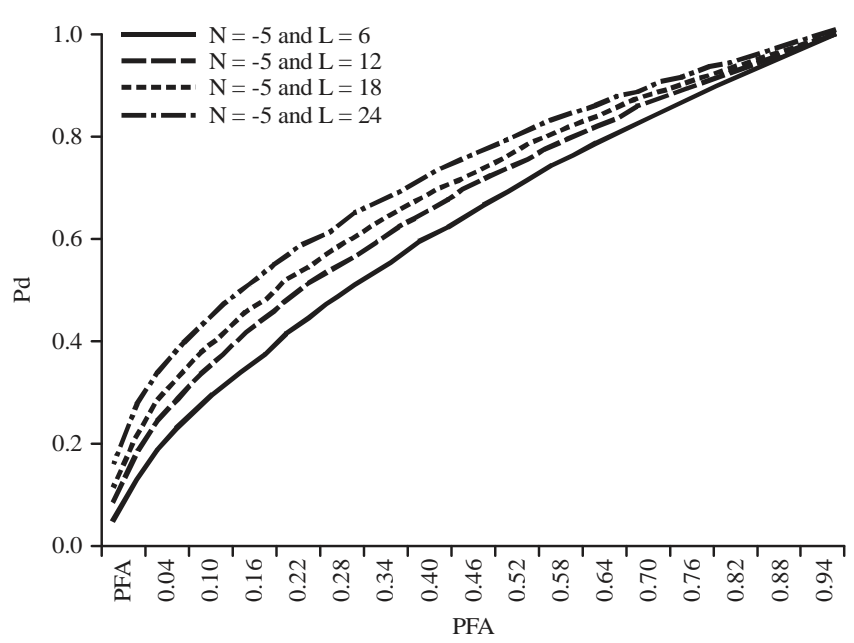

Fig. 8: ROC For various samples number and fixed SNR

consider the values, $-5,-10,-15$ for the SNR (Fig. 7). The energy detector performs well at moderate and high SNRs, it performs poorly at a low SNR. The three curves in question do not allow to decide whether the PU is present or absent, thus, creating a confusion which could cause interference to the licensed operator.

It is noticeably clear that ED's performance under the Rayleigh channel degrades for a low SNR. Many alternatives are possible To overcome this or at least improve it, we quote as an example, the increase in the number of samples (Chen, 2010), this can allow to have more clearly the signal Thus, we proceed by fix the SNR at low level with parallel support of increasing the number of samples (Fig. 8).

The different curves show a little improvement of detection, indeed to compensate the low level of SNR we increase the number of samples involved in the process of sounding the channel. We can also call on another metric that can show the trade-off between the false-alarm and detection, it is AUC (Area Under Curve) which is a compact and single value quantity which is a measure for the overall detection capability of a detector. It varies between $1 / 2$ and 1 .

As stated in (Barrett et al., 1998), the exact calculation of the AUC is difficult for realistic detection tasks. However, we note that, some research efforts, not necessarily deployed for wireless communications, mainly focus on bounds, asymptotic expansions or limiting value of AUCs.

However, increasing the number of samples will induce a high complexity and a sensing time, it will be preferable to study the relation between the probability of detection and the duration of listening of the channel. We also note that this will lead to the eminent phenomenon of increasing energy consumption in radio devices.

\section{CONCLUSION}

Cognitive radio is an optimal solution for improve spectral underutilization. We were interested in the Energy Detector (ED), tools among others covering the function of detection of white holes in the frequency spectrum. We did a spectrum detection scenario as part of the Rayleigh fading channel. It should be noted that our schema leads to interesting detection results in the field where the SNRs are high. Sadly, the detector fails to detect the primary user when the SNR level is a bit low. We thought to see what the introduction of a single parameter of fading, can bring improvement to our system. in this sense, the increase in the number of samples (Diversity parameter) is tested against a SNR set at a low value, it is clear that the detection improves a little but remains below the recommendations of the 80222 standard. It would be nice on the one hand to have ideas on the cost of this solution because it will introduce without a shadow of a doubt a calculation complexity and a listening time of the channel which according to the norm 802.22 does not exceeds 2 sec. On the other hand, at the level of the modulation step recoding techniques can be used to introduce redundancy into the signal. We plan to mitigate the weaknesses of this detector by using diversity combining and cooperative spectrum sensing techniques.

\section{REFERENCES}

Anonymous, 2019. Flat fading channel. MathWorks, Natick, Massachusetts, USA. https://fr.mathworks. com/matlabcentral/fileexchange/36620-flat-fadingchannel 
Atapattu, S., C. Tellambura and H. Jiang, 2009. Energy detection of primary signals over $\eta-\mu$ fading channels. Proceedings of the 2009 International Conference on Industrial and Information Systems (ICIIS), December 28-31, 2009, IEEE., Sri Lanka, pp: 118-122.

Atapattu, S., C. Tellambura and H. Jiang, 2011. Spectrum sensing via energy detector in low SNR. Proceedings of the 2011 IEEE International Conference on Communications (ICC), June 5-9, 2011, IEEE, Kyoto, Japan, pp: 1-5.

Atapattu, S., C. Tellambura and H. Jiang, 2014. Energy Detection for Spectrum Sensing in Cognitive Radio. Springer, New York, USA., ISBN: 978-1-4939-0493-8, Pages: 83.

Barbieri, A., A. Piemontese and G. Colavolpe, 2009. On the ARMA approximation for fading channels described by the Clarke model with applications to Kalman-based receivers. IEEE. Trans. Wireless Commun., 8: 535-540.

Barrett, H.H., C.K. Abbey and E. Clarkson, 1998. Objective assessment of image quality III. ROC metrics, ideal observers and likelihood-generating functions. J. Opt. Soc. Am. A., 15: 1520-1535.

Budiarjo, I., H. Nikookar and L.P. Ligthart, 2008. Cognitive radio modulation techniques. IEEE. Signal Process. Mag., 25: 24-34.

Cabric, D., S.M. Mishra and R.W. Brodersen, 2004. Implementation issues in spectrum sensing for cognitive radios. Proceedings of the 38th Asilomar Conference on Signals, Systems and Computers, November 7-10, 2004, Pacific Grove, CA., USA., pp: 772-776.

Chen, Y., 2010. Improved energy detector for random signals in Gaussian noise. IEEE. Trans. Wireless Commun., 9: 558-563.

De Alencar, R.T., M.D.A. Cavalcante, W.T.A. Lopes, W.J.L. de Queiroz and M.S. de Alencar, 2014. Modulation diversity effects in rayleigh fading multipath channels. Proceedings of the 2014 International Symposium on Telecommunications (ITS), August 17-22, 2014, IEEE., Sao Paulo, Brazil, pp: 1-5.

Du, Z., J. Cheng and N.C. Beaulieu, 2006. Accurate error-rate performance analysis of OFDM on frequency-selective Nakagami-m fading channels. IEEE. Trans. Commun., 54: 319-328.

FCC., 2002. Spectrum policy task force report. Federal Communications Commission, Washington, DC., USA.

Junior, R.A.D.S., R.A.A. de Souza and D.A. Guimaraes, 2013. Performance analysis of spectrum sensing techniques in nakagami and rice fading channels. Proceedings of the 31th Brazilian Telecommunication Symposium-SBrT2013, September 1-4, 2013, Fortaleza, Brazil, pp: 1-5.
Lee, S.H., D.C. Oh and Y.H. Lee, 2009. Hard decision combining-based cooperative spectrum sensing in cognitive radio systems. Proceedings of the 2009 International Conference on Wireless Communications and Mobile Computing: Connecting the World Wirelessly, Leipzig, Germany, June 21-24, 2009, ACM., New York, USA., pp: 906-910.

Mfeze, M. and E. Tonye, 2015. Comparative approach of Doppler spectra for fading channel modelling by the filtered white Gaussian noise method. Int. J. Comput. Sci. Telecommun., 6: 1-12.

Nallagonda, S., S. Suraparaju, S.D. Roy and S. Kundu, 2011. Performance of energy detection based spectrum sensing in fading channels. Proceedings of the 2nd International Conference on Computer and Communication Technology (ICCCT-2011), September 15-17, 2011, IEEE., Allahabad, India, pp: 575-580.

Sklar, B. and P.K. Ray, 2009. Digital Communications. 2nd Edn., Pearson Education, Delhi, India, ISBN:978-81-317-2092-9, pp: 168-236.

Sun, H., A. Nallanathan, C.X. Wang and Y. Chen, 2013. Wideband spectrum sensing for cognitive radio networks: A survey. IEEE. Wireless Commun., 20: 74-81.

Tlili, F., A. Bouallegue and A. Glavieux, 1999. Channel coding input for OFDM-DPSK transmission on rice and rayleigh channels. Dix-Septième Colloque GRETSI, Vannes, Septembre 13-17, 1999, pp: 861-864.

Venkateswarlu, S. and J.K.R. Sastry, 2014. Justification of Rayleigh faded channel for data transmission in wireless environment. Int. J. Eng. Trends Technol., 14: 166-168.

Yelizati, J., 2016. Matlab-based error analysis of PSK and QAM. https://www.academia.edu/35860362/ReportMATLAB-BASED_ERROR_ANALYSIS_OF_ PSK_AND_QAM

Young, D.J. and N.C. Beaulieu, 2000. The generation of correlated Rayleigh random variates by inverse discrete Fourier transform. IEEE. Trans. Commun., 48: 1114-1127.

Yucek, T. and H. Arslan, 2009. A survey of spectrum sensing algorithms for cognitive radio applications. IEEE. Commun. Surv. Tutorials, 11: 116-130.

Zeng, Y., Y.C. Liang, A.T. Hoang and R. Zhang, 2010. A review on spectrum sensing for cognitive radio: Challenges and solutions. EURASIP J. Adv. Signal Process., Vol. 2010. 10.1155/2010/381465. 